\title{
Analysis of group paging with pre-backoff
}

\author{
Ruki Harwahyu ${ }^{1}$, Xian Wang ${ }^{2}$, Riri Fitri Sari ${ }^{3}$ and Ray-Guang Cheng ${ }^{1 *}$
}

\begin{abstract}
Group paging can simultaneously activate hundreds of user equipments (UEs) using a single paging message. Upon receiving the group paging message, all UEs should immediately transmit their paging response messages through the random access channels (RACHs). Simultaneous channel access from a huge group of UEs may result in severe collisions of the RACHs during a very short period of time. In this paper, we propose a pre-backoff method to reduce the collision probability of random access requests. We develop an analytical model to investigate the performance and optimize the setting of the pre-backoff method. The accuracy of the analytical model is verified through computer simulation. Results show that the proposed pre-backoff method can effectively enhance the performance of group paging.
\end{abstract}

Keywords: Group paging; Long term evolution (LTE); Pre-backoff; Random access

\section{Introduction}

Machine-type communication (MTC) is one of the most important services for next generation networks. 3GPP, IEEE and other international organizations adopt their technologies to the emerging market of the MTC [1]. Mass machine devices that simultaneously access the network may result in heavy collisions and thus, degrade the network performance [1,2]. In 3GPP [3], a concept of group paging has been introduced to alleviate the heavy collision problem in the random access channels (RACHs) of the cellular networks. However, no normative specification work on group issues took place. It may restart again in the next 3GPP Release 13. In group paging, the base station, which is known as an evolved-NodeB (eNB) in 3GPP, can assign a number of machine devices to form a paging group. The eNB can then simultaneously activate the group of machine devices by sending a single group paging message. Upon receiving the group paging message, all machine devices belonging to the paging group should immediately perform the random access (RA) procedure to access the network during a period of time, which is referred as a group paging interval herein [4].

The concept of grouping is also adopted by IEEE 802.11ah [1] to simplify operation with a huge number of associated machine devices. IEEE 802.11ah developed

\footnotetext{
*Correspondence: crg@mail.ntust.edu.tw

${ }^{1}$ Department of Electrical, Electronics and Computer Engineering, National Taiwan University of Science and Technology (NTUST), Taipei 106, Taiwan
} Full list of author information is available at the end of the article a restricted access window (RAW) mechanism to limit the set of machine devices accessing the channel and to spread their access attempts over a long period of time. In other words, RAW divides stations into groups, splits the channel into slots, and assigns each slot to a group. The machine devices can only transmit in the slot assigned to their group [1]. In IEEE 802.11ah, the base station, which is known as an access point (AP), may wake up a huge number of machine devices by setting the association identity bits in a traffic indication map (TIM) element carried by the beacon frame [1].

The related works of group paging are briefly summarized as below. The performance analysis of group paging via computer simulations was first addressed in [5]. The first analytical model of a simplified group paging without random backoff was first presented in [6]. The model is then completed and extended in [4] to analyze the performance of group paging by considering all the implementation constraints of the RA procedure specified in LTE networks. A group-based time control mechanism was presented in [7] to deal with radio access network overload problem in a femtocell-based MTC network. A priority grouping scheme is presented in [8] to deal with the $\mathrm{RACH}$ collisions by assigning priority-dependent backoff parameters. In [9], the authors proposed a twolevel device partitioning scheme to deal with the RACH overload problem by limiting the number of contending machine devices. In [10], the authors proposed to alleviate the RACH collision problem by assigning dedicated

\section{望 Springer}


preamble and RA slot for each UE. Our previous work in [2] introduces consecutive group paging, which provides several group paging intervals consecutively for a group. Normal group paging is performed in each interval, and failing UEs from the previous interval are paged again in the successive interval. Compared with the original group paging, this method slightly increases the success probability at the cost of increased access delay.

In group paging, heavy RACHs collisions are found at the beginning of the group paging interval due to the simultaneous channel accesses from the whole group of machine devices. A randomized paging response method has been proposed in 3GPP SA2 to deal with the heavy collision problem but without any follow-up work. In [11], the authors proposed a pre-backoff scheme to relief the heavy collision problem. The pre-backoff scheme enforces each machine device to perform a random backoff before its first preamble transmission. A preliminary study of the pre-backoff scheme in group paging via computer simulations was presented in [12]. The results showed that the pre-backoff scheme may properly reduce the heavy collision problem. However, it is not clear how to determine the optimum pre-backoff window size under a given group size.

This paper presents an analytical model to analyze the performance of pre-backoff scheme for group paging. The optimal setting for the pre-backoff window is then derived. The accuracy of the analytical model was then verified through computer simulations. Instead of performing paging consecutively, the method proposed in this paper spreads the first preamble transmission by using a pre-backoff mechanism to increase the success probability with lower access delay compared with the original group paging. The rest of the article is organized as follows. Section 2 summarizes the system model. The concept of group paging with pre-backoff is briefly introduced. The analytical model of group paging with prebackoff is then elaborated. Simulation results are shown in Section 3. Section 4 draws the conclusion.

\section{System model}

\subsection{Random access procedure in group paging}

The random access model considered in the paper follows the LTE random access procedure standard. In LTE, time is divided into fix-length radio frames. Each radio frame consists of 20 sub-frames. One or more sub-frames in a radio frame are reserved for UEs to perform random access. These special sub-frames are referred to as RA slots herein. Each RA slot contains $R$ RAOs. In group paging, a group paging interval is reserved for a group of $M$ UEs. There are $I_{\max }$ RA slots in a group paging interval. Let $T_{R A \_R E P}$ be the number of sub-frames between successive RA slots. All symbols used herein are summarized in Table 1.
Table 1 System parameters and symbols used in the equations

\begin{tabular}{|c|c|}
\hline Notation & Definition \\
\hline$R$ & Total random access opportunities (RAOs) \\
\hline M & Total UE in the paged group \\
\hline$I_{\max }$ & Total RA slots in a group paging interval \\
\hline$T_{R A \_R E P}$ & Interval between successive RA slots (unit: sub-frame) \\
\hline$T_{\text {RAR }}$ & $\begin{array}{l}\text { Processing time required by eNB to detect transmitted } \\
\text { preambles (unit: sub-frame) }\end{array}$ \\
\hline$W_{\text {RAR }}$ & Window of random access reply (RAR) (unit: sub-frame) \\
\hline$N_{R A R}$ & $\begin{array}{l}\text { Maximum number of acknowledgement that can be } \\
\text { carried in a RAR message }\end{array}$ \\
\hline NUL & Maximum acknowledged UE in one RAR window \\
\hline$W_{B O}$ & Window backoff (unit: sub-frame) \\
\hline NPTmax & $\begin{array}{l}\text { Maximum attempt by each UE to transmit preamble in } \\
\text { one group paging interval }\end{array}$ \\
\hline$T_{C R}$ & Contention resolution timer (unit: sub-frame) \\
\hline$T_{H A R Q}$ & $\begin{array}{l}\text { Interval for receiving HARQ acknowledgment } \\
\text { (unit: sub-frame) }\end{array}$ \\
\hline$T_{M 3}$ & Gap for Msg3 retransmission (unit: sub-frame) \\
\hline$N_{H A R Q}$ & Maximum attempt of $\mathrm{HARQ}$ transmission of Msg3 and Msg4 \\
\hline$T_{A \_M 4}$ & Gap of monitor Msg4 (unit: sub-frame) \\
\hline$T_{M 4}$ & Gap for Msg4 retransmission (unit: sub-frame) \\
\hline$W_{P B O}$ & Window of pre-backoff (unit: sub-frame) \\
\hline$M_{i}$ & $\begin{array}{l}\text { Total number of UE that transmit their preambles in the ith } \\
\text { RA slot }\end{array}$ \\
\hline$M_{i}[n]$ & $\begin{array}{l}\text { Number of contending UE that transmit the } n \text {th preamble } \\
\text { at the ith RA slot }\end{array}$ \\
\hline$M_{i, S}[n]$ & $\begin{array}{l}\text { Portion of } M_{i}[n] \text { that successfully complete its preamble } \\
\text { transmission }\end{array}$ \\
\hline$M_{i, F}[n]$ & Portion of $M_{i}[n]$ that fail in its preamble transmission \\
\hline$P_{p}$ & Probability of an UE to correctly receive paging message \\
\hline$\alpha_{k, i}$ & $\begin{array}{l}\text { The portion of backoff interval of the } k \text { th } R A \text { slot } \\
\left(K_{\min }=k=K_{\max }<i\right) \text { that overlap with the transmission } \\
\text { interval of the ith RA slot }\end{array}$ \\
\hline$P_{n}$ & $\begin{array}{l}\text { The detection probability of the } n \text {th preamble transmission } \\
\text { due to power-ramping effect }\end{array}$ \\
\hline$P_{C}$ & $\begin{array}{l}\text { The ratio between the total number of collided preamble } \\
\text { and to all allocated preamble }\end{array}$ \\
\hline$P_{S}$ & $\begin{array}{l}\text { The ratio between total UE that successfully access the } \\
\text { network and the total number of UE in the paged group }\end{array}$ \\
\hline$\overline{D_{A}}$ & $\begin{array}{l}\text { The total access delay for all successfully accessed UEs } \\
\text { normalized by the total successfully accessed UEs }\end{array}$ \\
\hline$T_{i}$ & $\begin{array}{l}\text { The access delay for successfully accessed UEs from the } \\
\text { transmitting preamble at the ith RA slot }\end{array}$ \\
\hline$\overline{T_{M S G}}$ & $\begin{array}{l}\text { The average time for one UE to finish the message-part } \\
\text { transmission }\end{array}$ \\
\hline$D_{f}$ & HARQ retransmission probability for Msg3 and Msg4 \\
\hline
\end{tabular}

The whole RA procedure assumed in this paper is shown in Figure 1. After being paged, all UEs randomly transmit one of the $R$ preambles at the first RA 


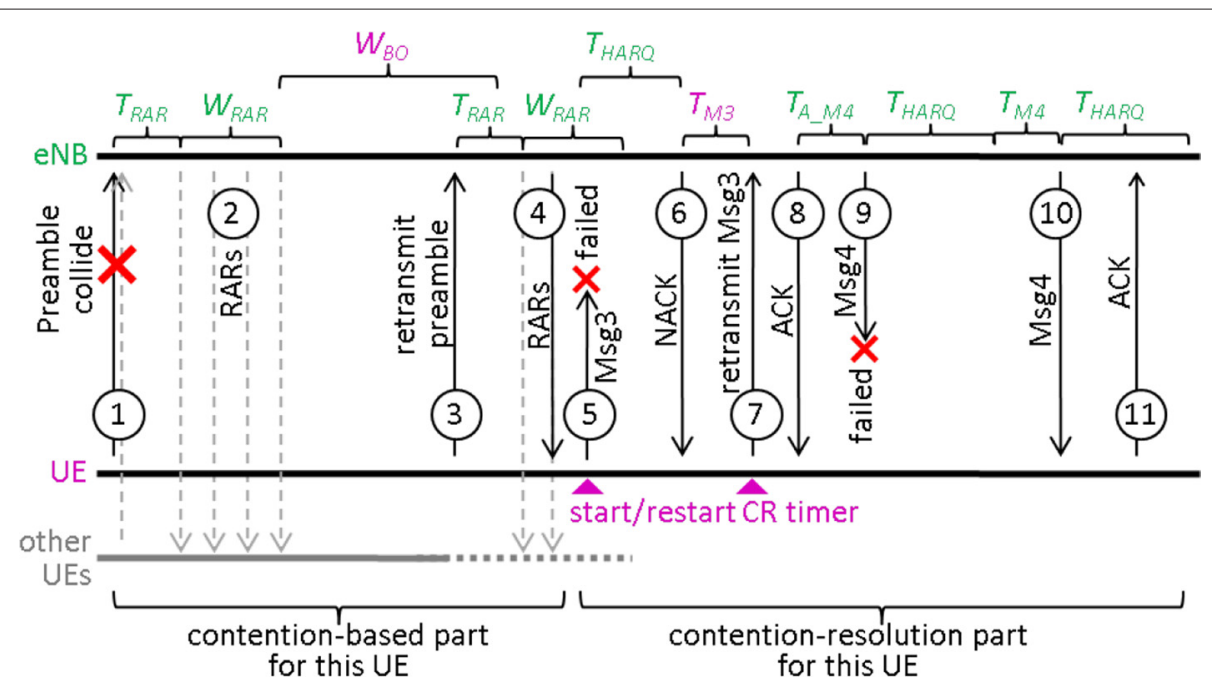

Figure 1 The steps in an RA procedure.

slot (step 1). $T_{R A R}$ is the processing time (unit: subframe) required by an eNB to detect the transmitted preambles. The eNB subsequently broadcasts random access response (RAR) messages to inform which UEs are acknowledged and granted for resource (step 2). $W_{R A R}$ is the RAR window (unit: sub-frame), i.e., the time interval for the eNB to transmit RAR messages. Each RAR message can carry up to $N_{R A R}$ acknowledgements. The maximum number of UEs that can be acknowledged during an RAR window, which is denoted by $N_{U L}$, is $W_{R A R} N_{R A R}$.

Preamble transmission may fail due to some conditions, i.e., not-detected (due to underpowered), collided (detected but not unique among other UEs which transmit preamble at the same RA slot) or not collided but not selected, since the eNB can only acknowledge $N_{U L}$ preambles at a $W_{R A R}$. If an UE does not receive RAR in $\left(T_{R A R}+W_{R A R}\right)$ sub-frames after its preamble transmission, it performs backoff with the window of $W_{B O}$ and then transmits the new preamble with an increased power at the immediate RA slot (step 3). UE can only attempt to transmit preamble up to $N_{P \text { Tmax }}$ times in one group paging interval.

After receiving RAR, the UE sends Msg3 at the next sub-frame (step 5). Each time the UE sends Msg3, it counts down $T_{C R}$ sub-frames as a contention-resolution timer. The reason this timer is employed is to detect the collision of Msg3. In some cases, the eNB may successfully decode one preamble transmitted by multiple UEs because of constructive interference and then reply with a RAR accordingly, assuming that the preamble is transmitted by one UE instead of two. These two UEs will transmit their own Msg3 on the same resource and realize the random access failure after the $T_{C R}$ expires without receiving anything from eNB.
The eNB sends NACK if it does not receive Msg3 in $T_{\text {HARQ }}$ sub-frames after it sends RAR (step 6) or ACK otherwise (step 8). If UE receives NACK, it retransmits Msg3 in $T_{M 3}$ sub-frames later (step 7). UE goes back to preamble transmission stage if Msg3 fails $N_{H A R Q}$ times. The eNB transmits Msg4 in $T_{A_{-} M 4}$ sub-frames after it sends Msg3's ACK (step 9). Subsequently, eNB waits for Msg4's ACK in $T_{\text {HARQ }}$ sub-frames. If Msg4's ACK is not received, eNB retransmits Msg4 in $T_{M 4}$ sub-frames later (step 10). The UE goes back to the preamble transmission stage if it still fails to receive Msg4 $N_{H A R Q}$ times (eNB can send Msg4 up to $N_{H A R Q}$ times). After receiving Msg4, the UE immediately send the Msg4's ACK to finish the RA procedure (step 11).

\subsection{Pre-backoff for group paging}

This paper suggests the use of pre-backoff to enhance the performance of original group paging considered in [4]. In group paging with pre-backoff, each UE should perform pre-backoff for its first transmission and follow the standard LTE random access procedure for the retransmissions. The pre-backoff timer is uniformly chosen in the range 0 to $W_{P B O}$.

The performance of group paging with pre-backoff during a group paging interval of $I_{\text {max }}$ th RA slots is investigated. The group paging interval starts from the first RA slot and ends at the $I_{\text {max }}$ th RA slot. The pre-backoff may postpone the transmission time of the first preamble up to the pre-backoff window, $W_{P B O}$. The group paging interval is given in (1).

$$
I_{\text {max }}=\left\lceil\frac{W_{P B O}}{T_{R A \_R E P}}\right\rceil+1+\left(N_{P T \max }-1\right)\left\lceil\frac{T_{R A R}+W_{R A R}+W_{B O}}{T_{R A \_R E P}}\right\rceil .
$$


Let $M_{i, S}[n]$ and $M_{i, F}[n]$ be the number of successful and failed UEs that transmit the $n$th preambles at the $i$ th RA slot, respectively; $M_{i}[n]$ be the total number of UEs that transmit the preamble in the $i$ th RA slot; and $P_{P}$ be the probability of an UE to correctly receive paging message. Upon receiving the paging message, $P_{P} M$ UEs will immediately perform a uniform backoff before transmitting their first preamble. The total number of UEs that transmit their first preamble in the $i$ th RA slot, $M_{i}[1]$, is derived in (2).

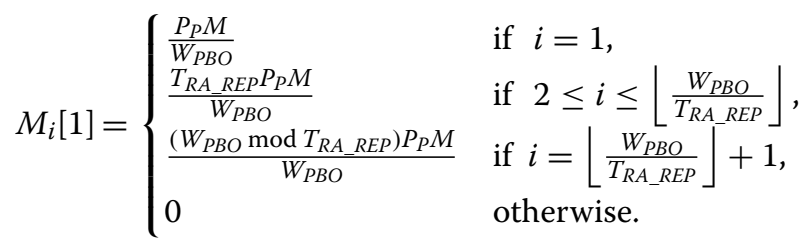

RA attempts in the first RA slot and the other RA slots are investigated separately, since there is no preamble retransmission in the first RA slot. The total number of successful UEs, $M_{1, S}[n]$, and failed UEs, $M_{1, F}[n]$, from the first RA slot is given in (3) and (4), respectively. Note that at the initial condition of $i=1, M_{1}[n]=0$ for $n \neq 1$.

$$
M_{1, S}[n]= \begin{cases}N_{U L} & \text { if } n=1 \text { and } M_{i}[1] e^{-\frac{M_{i}[1]}{R}} p_{1} \geq N_{U L}, \\ M_{i}[1] e^{-\frac{M_{i}[1]}{R}} p_{1} & \text { if } n=1 \text { and } M_{i}[1] e^{-\frac{M_{i}[1]}{R}} p_{1}<N_{U L}, \\ 0 & \text { if } n \neq 1 ;\end{cases}
$$

$$
M_{1, F}[n]= \begin{cases}M_{i}[1]-N_{U L} & \text { if } n=1 \text { and } M_{i}[1] e^{-\frac{M_{i}(1)}{K}} p_{1} \geq N_{U L} \\ M_{i}[1]\left(1-e^{-\frac{M_{i}(11}{R}} p_{1}\right) & \text { if } n=1 \text { and } M_{i}[1] e^{-\frac{M_{i}[1]}{R}} p_{1}<N_{U L}, \\ 0 & \text { if } n \neq 1 .\end{cases}
$$

where $N_{U L}$ is the maximal total number of UEs that can be acknowledged in one response window and $p_{n}$ is the detection probability of the $n$th preamble transmission due to power-ramping effect [6], where $p_{n}=1-\left(1 / e^{n}\right)$. Failed UEs in the first RA slot will perform backoff and retransmit again in the following RA slots. The total number of successful and failed UEs for each attempt from each RA slot, $M_{i, S}[n]$ and $M_{i, F}[n]$, can be recursively obtained from (5) and (6), respectively.

$$
M_{i, S}[n]= \begin{cases}M_{i}[n] e^{-\frac{M_{i}}{R}} P_{n} & \text { if } \sum_{n=1}^{N_{P T \max }} M_{i}[n] e^{-\frac{M_{i}}{R}} p_{n} \leq N_{U L}, \\ \frac{M_{i}[n] e^{-\frac{M_{i}}{R}} P_{n}}{\sum_{n=1}^{N_{\text {PTax }}} M_{i}[n] e^{-\frac{M_{i}}{R}} p_{n}} & \text { otherwise; } \\ n_{n=1} & \end{cases}
$$

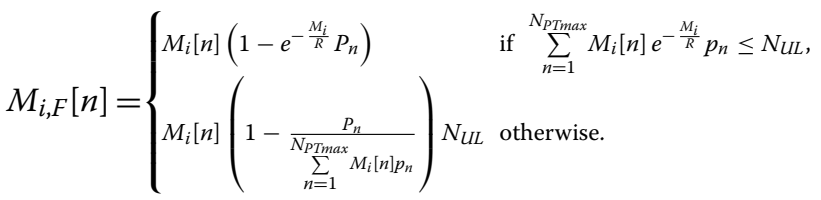

$$
\begin{aligned}
& M_{i}[n] \approx \sum_{k=K_{\min }}^{K_{\max }} \alpha_{k, i} M_{k, F}[n-1] \quad \text { if } n>1 .
\end{aligned}
$$

The total number of UEs that conduct their $n$th RA attempt in the $i$ th RA slot, $M_{i}[n]$, can be approximated by (7) for $n>1 . M_{k, F}[n-1]$ in (7) represents the UEs that transmit their $(n-1)$ th preambles at the $k$ th RA slot but fail. $\alpha_{k, i}$ of these failed UEs conduct retransmission in the $i$ th RA slot. $K_{\min }$ and $K_{\max }$ denote the minimal and maximal value of $k$, respectively.

The transition probability, $\alpha_{k, i}$, its upper bound, $K_{\max }$, and lower bound, $K_{\min }$, in (7) can be derived based on the timing diagram given in Figure 2. $\alpha_{k, i}, K_{\max }$, and $K_{\min }$ are given in (8), (9), and (10), respectively. The UEs with failed preamble transmission at the $k$ th RA slot may retransmit a new preamble at the $i$ th RA slot only if the backoff interval of the $k$ th RA slot is overlapped with the transmission interval of the $i$ th RA slot. Therefore, $\alpha_{k, i}$ is the portion of the backoff interval of the $k$ th RA slot which overlaps with the transmission interval of the $i$ th RA slot (with $k<i$ ). In Figure 2, UEs which transmit their preambles at the $k$ th RA slot at time $(k-1) T_{R A \_R E P}$ will recognize their RA failure after $\left(T_{R A R}+W_{R A R}\right)$ sub-frames. Each failed UE starts the backoff at time $(k-1) T_{R A \_R E P}+\left(T_{R A R}+W_{R A R}\right)+1$. Therefore, the backoff interval of the $k$ th RA slot starts from $(k-1) T+{ }_{R A \_R E P}+\left(T_{R A R}+W_{R A R}\right)+1$ and ends at $(k-1) T_{R A \_R E P}+\left(T_{R A R}+W_{R A R}\right)+W_{B O}$. The UEs transmit their preambles at $i$ th RA slot if their backoff expires between the $(i-1)$ th and the $i$ th RA slot. Therefore, the transmission interval of the $i$ th $R A$ slot is $\left[(i-1) T_{R A} R E P+\right.$ $\left.1(i-1) T_{R A \_R E P}\right]$.

$K_{\min }$ is obtained when the right boundary of the $k$ th RA slot backoff interval reaches the left boundary of $i$ th RA slot transmission interval, which is $\left(K_{\text {min }}-1\right) T_{R A \_R E P}+$ $T_{R A R}+W_{R A R}+W_{B O} \geq 1+(i-2) T_{R A \_R E P}$. Therefore, $K_{\text {min }}$ is expressed as

$$
K_{\text {min }}=\left\lceil(i-1)+\frac{1-\left(T_{R A R}+W_{R A R}+W_{B O}\right)}{T_{R A \_R E P}}\right\rceil .
$$

The maximal value of $k\left(K_{\max }\right)$ is obtained when the left boundary of the $k$ th RA slot backoff interval exceeds the right side boundary of the $i$ th RA slot transmission interval, which is $\left(K_{\max }-1\right) T_{R A \_R E P}+T_{R A R}+W_{R A R}+$ $1 \leq(i-2) T_{R A \_R E P}$. Hence,

$$
K_{\max }=\left\lfloor i-\frac{1+\left(T_{R A R}+W_{R A R}\right)}{T_{R A \_R E P}}\right\rfloor .
$$




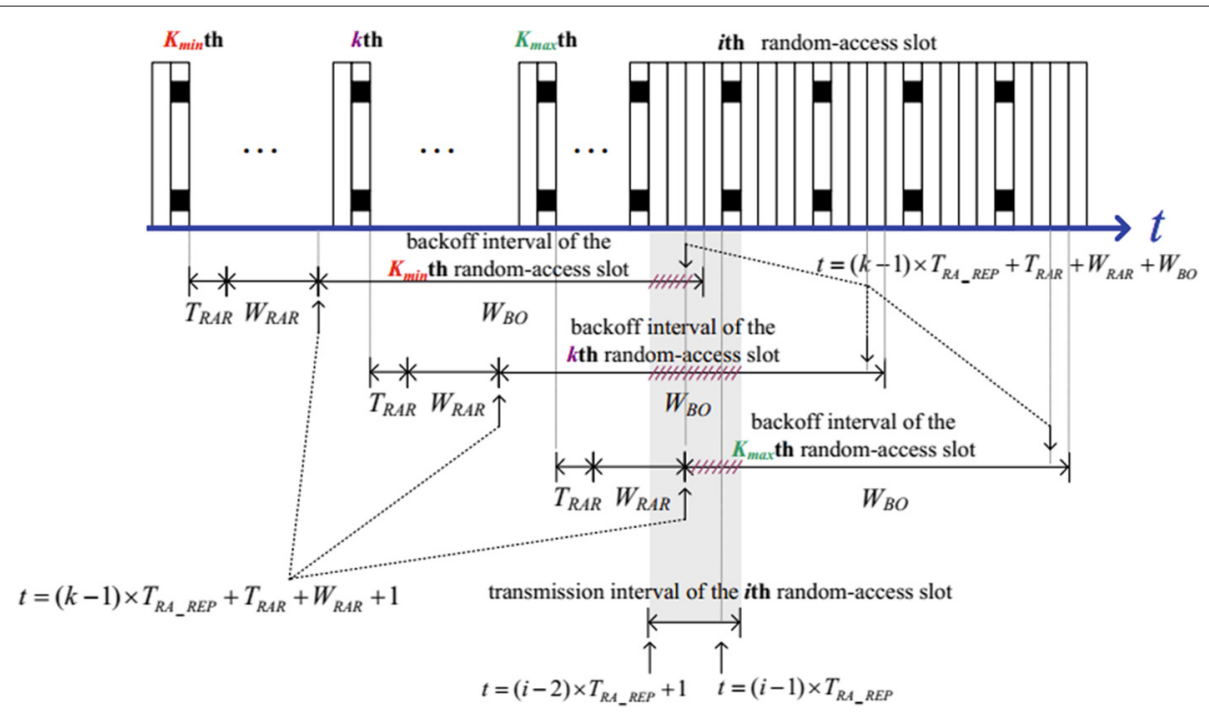

Figure 2 Timing diagram which shows the preamble retransmission.

$\alpha_{k, i}$ can be determined based on $k$ in the three cases shown in the lower part of Figure 2. In the first case, the right boundary of the backoff interval is within the transmission interval, i.e., $1+(i-2) T_{R A \_R E P} \leq(k-1) T_{R A \_R E P}+$ $T_{R A R}+W_{R A R}+W_{B O} \leq(i-1) T_{R A \_R E P}$. In this case, $K_{\text {min }} \leq k \leq i-\left(\left(T_{R A R}+W_{R A R}+W_{B O}\right) / T_{R A \_R E P}\right)$ and the overlapped region starts from the left boundary of the transmission interval and ends at the right boundary of the backoff interval. In the second case, the transmission interval is fully overlapped with the backoff interval. Therefore, the length of the overlapped region is $T_{R A} R E P$. In the third case, the left boundary of the backoff interval is within the transmission interval $1+(i-2) T_{R A \_R E P} \leq$ $(k-1) T_{R A \_R E P}+T_{R A R}+W_{R A R}+1 \leq(i-1) T_{R A \_R E P}$. In this case, $(i-1)-\left(\left(T_{R A R}+W_{R A R}\right) / T_{R A \_R E P}\right) \leq$ $k \leq K_{\max }$ and the overlapped region starts from the left boundary of the backoff interval and ends at the right boundary of the transmission interval. $\alpha_{k, i}$ is the ratio of the overlapped region to the backoff interval and is expressed as

$$
\alpha_{k, i}=\left\{\begin{array}{l}
\frac{(k-1) T_{R A \_R E P}+T_{R A R}+W_{R A R}+W_{B O}-(i-2) T_{R A \_R E P}}{W_{B O}} \\
\quad \text { if } K_{\text {min }} \leq k \leq i-\frac{T_{R A R}+W_{R A R}+W_{B O}}{T_{R A \_R E P}}, \\
\frac{T_{R A \_R E P}}{W_{B O}} \\
\text { if } i-\frac{T_{R A R}+W_{R A R}+W_{B O}}{T_{R A} R E P}<k<i-1-\frac{T_{R A R}+W_{R A R}}{T_{R A \_} R E P}, \\
\left.\frac{(i-1) T_{R A \_R E P}-\left((k-1) T_{R A} R E P\right.}{W_{B O}}+T_{R A R}+W_{R A R}\right) \\
\quad \text { if } i-1-\frac{T_{R A R}+W_{R A R}}{T_{R A \_R E P}} \leq k \leq K_{\text {max }}, \\
0 \quad \text { otherwise. }
\end{array}\right.
$$

\subsection{Performance metrics}

The performance metrics are derived to estimate the collision probability, $P_{C}$, the successful access probability, $P_{S}$, and average access delay, $\overline{D_{A}} \cdot P_{C}$ is the ratio of the total number of collided preambles to the entire allocated preambles. $P_{S}$ is the ratio of total UE that have successfully accessed the network to the total number of UE in the paged group. $\overline{D_{A}}$ is the total access delay for all successfully accessed UEs normalized by the total number of successfully accessed UEs. $P_{C}, P_{S}$, and $\overline{D_{A}}$ are given in (11), (12), and (13), respectively.

$$
\begin{aligned}
& P_{C}=\frac{\sum_{i=1}^{I_{\max }}\left(R-M_{i} e^{-\frac{M_{i}}{R}}-R e^{-\frac{M_{i}}{R}}\right)}{I_{\max } R}, \\
& P_{S}=\frac{\sum_{i=1}^{I_{\max } N_{P \max }} \sum_{n=1}^{M_{\text {max }}} M_{i, S}[n]}{M}, \\
& \overline{D_{A}}=\frac{\sum_{i=1}^{I_{\max } N_{P \max }} \sum_{n=1}^{I_{\max } N_{P \max }} M_{i, S}[n] T_{i}}{\sum_{i=1}^{\sum_{P=1}} M_{n=1}[n]},
\end{aligned}
$$

where $T_{i}$ is the access delay for the successfully accessed UEs from the transmitting preamble at the $i$ th RA slot, which can be obtained from (14).

$$
T_{i}=(i-1) T_{R A \_R E P}+T_{R A R}+W_{R A R}+\overline{T_{M S G}} .
$$

In (14), $\overline{T_{M S G}}$ is the average time required by an UE to finish the message-part transmission. $\overline{T_{M S G}}$ is the time used to transmit $u$ Msg3 and $v$ Msg4 and is given in (15). 


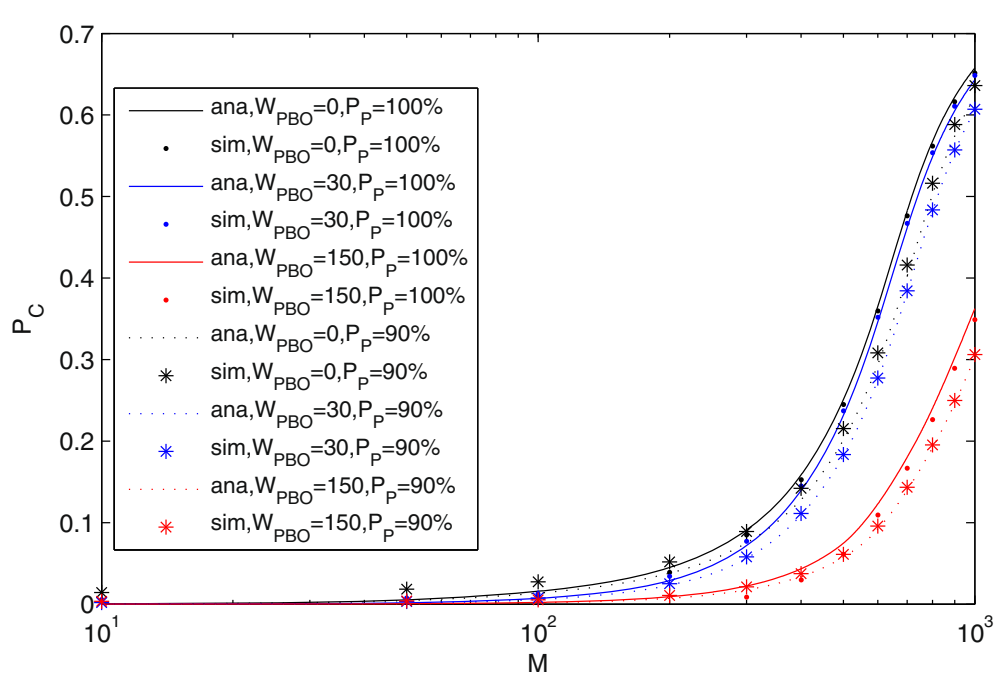

Figure 3 Collision probability, $P_{C}$.

Msg3 and Msg4 can be transmitted up to $N_{\text {HARQ }}$ times. Msg3 is sent directly after the UE receives RAR message while Msg4 is sent $T_{A_{-} M 4}$ sub-frames after eNB sends the Msg3's ACK. Each of Msg3's and Msg4's transmission should be followed by ACK within $T_{H A R Q}$ sub-frames; otherwise, they should be retransmitted. Msg3's retransmission is conducted after $T_{M 3}$ sub-frames and Msg4's is conducted after $T_{M 4}$ sub-frames. Hence, $\overline{T_{M S G}}$ can be expressed as

$$
\begin{aligned}
\overline{T_{M S G}}= & \sum_{u=1}^{N_{\text {HARQ }}} \sum_{v=1}^{N_{\text {HARQ }}} P_{f}^{u+v-2}\left(1-P_{f}\right)^{2} \times \\
& \left((u-1) T_{M 3}+(v-1) T_{M 4}+(u+v) T_{\text {HARQ }}+T_{A_{-} M 4}+1\right) .
\end{aligned}
$$

Note that higher $P_{S}$, lower $P_{C}$, or lower $\overline{D_{A}}$ are preferred. Our proposed method of pre-backoff is used to spread the first preamble transmissions. An optimal value of $W_{P B O}$ decreases $P_{C}$ and thus increases $P_{S}$. However, further increase in $W_{P B O}$ might decrease $P_{S}$ since a too large $W_{P B O}$ (relative to $M$ ) reserves longer time for the first RA attempt and reduces the opportunity for retransmission. Hence, the optimal $W_{P B O}$ for a given value of $M$ is the smallest value of $W_{P B O}$ that yields the highest $P_{S}$. The optimal $W_{P B O}$ here is effective for a delay-tolerant application since it might introduce an additional average access delay to reach the highest $P_{S}$. For an optimal value of $W_{P B O}$, the corresponding $N_{P T \text { Tmax }}$ can then be determined based on the $W_{P B O}$ using (1). In the implementation, the

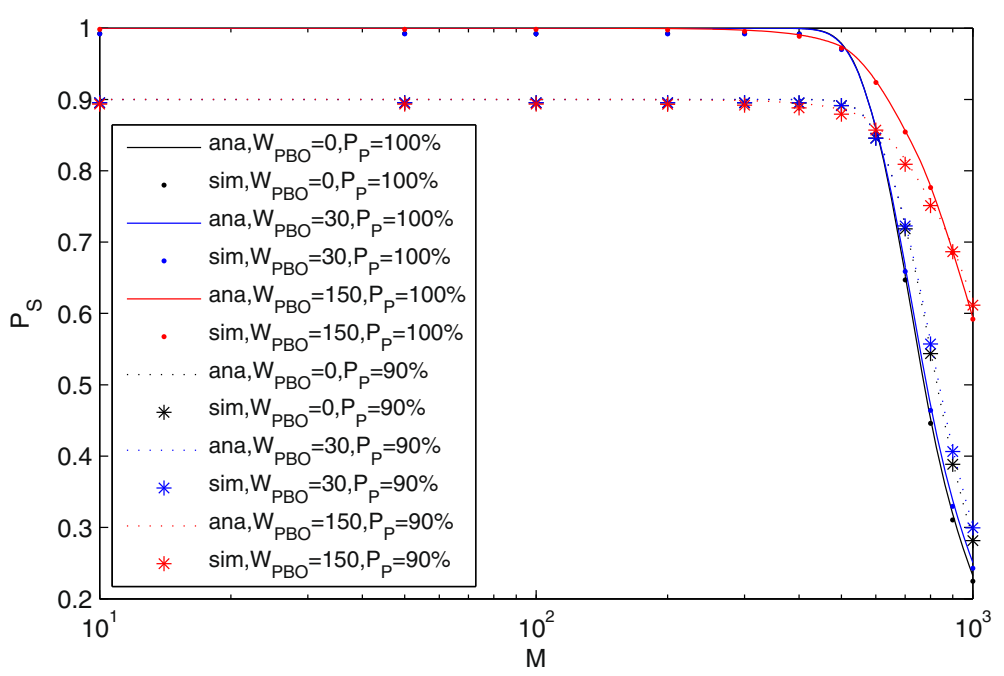

Figure 4 Access success probability, $P_{s}$. 


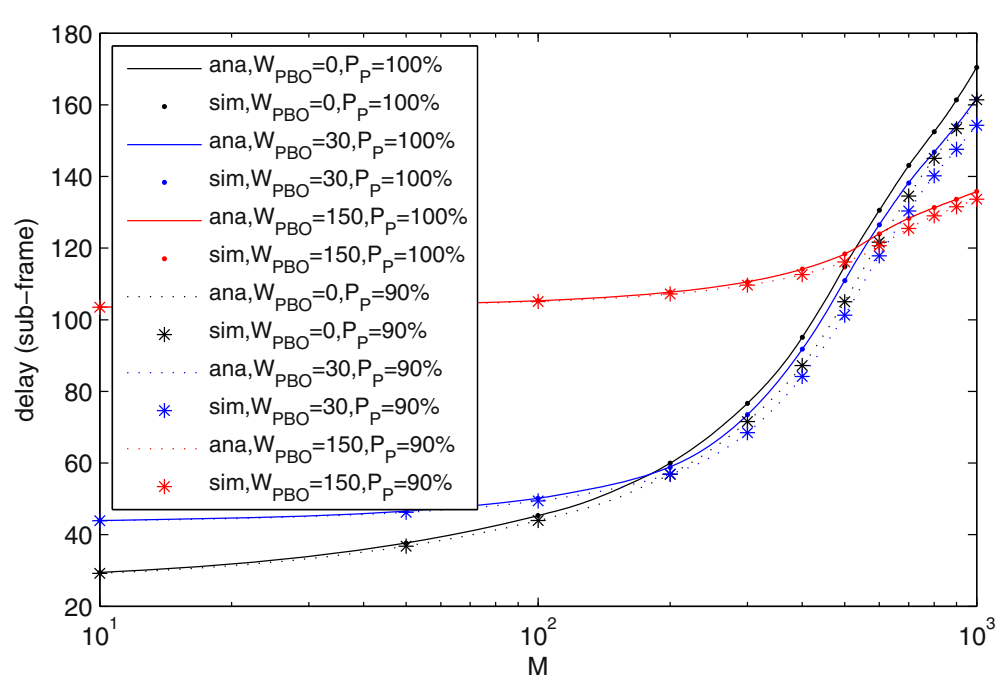

Figure 5 Average access delay, $\overline{D_{A}}$.

eNB can carry the optimal $W_{P B O}$ and its corresponding $N_{\text {PTmax }}$ along with the group paging message.

\section{Results and discussion}

The proposed analytical model is verified through computer-based simulation. Currently, there is no proper scenario that can be used to compare the performance of push-based and pull-based RAN overload control schemes. Hence, we did not compare group paging with the other push-based schemes. The analytical results are shown in line while the numerical results are shown in point, with each point representing the average value of $10^{7}$ samples.
Five values of $W_{P B O}$ were considered in the simulations of Figures 3, 4, 5 and 6 . The $N_{P \text { Tmax }}$ is adjusted for each $W_{P B O}$ according to (1) to ensure common $I_{\max }$ for a fair comparison. Table 2 shows the setting of $W_{P B O}$ and $N_{P \text { Tmax }}$. In Figures 3, 4 and 5, Cases I and II, and $P_{P}$ of $100 \%$ and $90 \%$ are examined. In Figure 6, Cases II to V and $P_{P}$ of $100 \%$ are examined. The remaining parameters are $I_{\max }=55, R=54, N_{U L}=15, T_{R A \_R E P}=5, W_{R A R}=$ $5, N_{H A R Q}=5, T_{R A R}=2, T_{H A R Q}=4, T_{M 3}=4, T_{A_{-} M 4}=$ 1 , and $T_{M 4}=1$.

A group size of 10 to 1,000 (i.e., $0 \leq M \leq 1,000$ ) is considered during the simulation. Figures 3,4 , and 5 depict $P_{C}, P_{S}$, and $\overline{D_{A}}$, respectively. Overall, the results

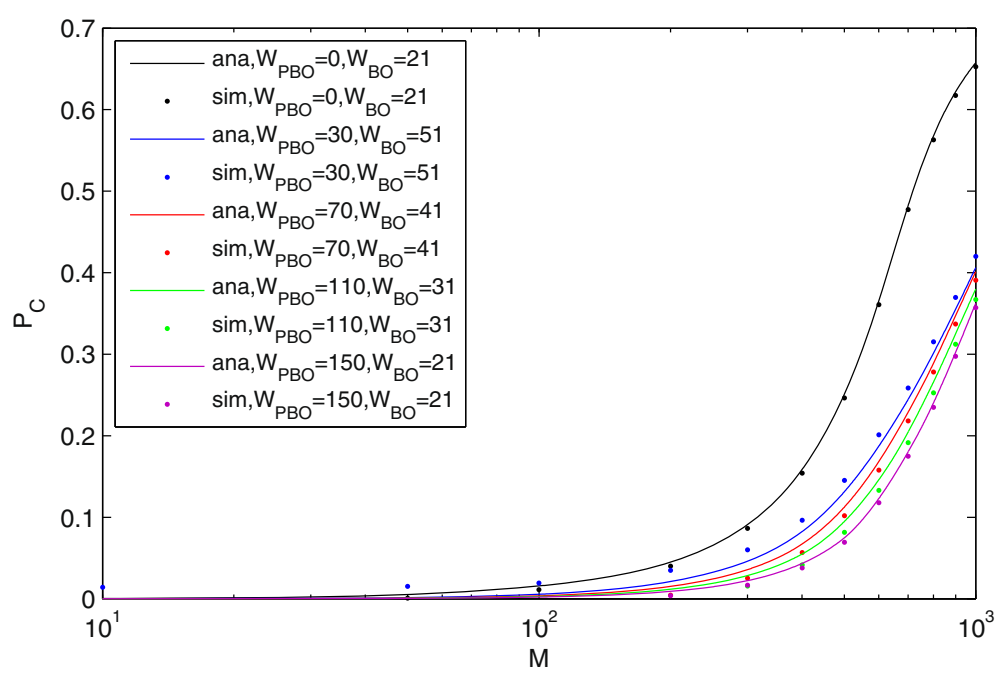

Figure 6 Collision probability, $P_{C}$. 
Table 2 Parameter setting of $W_{P B O}$ and $N_{P T \max }$

\begin{tabular}{lccc}
\hline Cases & $\boldsymbol{W}_{\boldsymbol{P B O}}$ & $\boldsymbol{N}_{\boldsymbol{P T m a x}}$ & $\boldsymbol{W}_{\boldsymbol{B O}}$ \\
\hline Original group paging case & 0 & 10 & 21 \\
Pre-backoff (Case I) & 30 & 9 & 21 \\
Pre-backoff (Case II) & 150 & 5 & 21 \\
Pre-backoff (Case III) & 30 & 5 & 51 \\
Pre-backoff (Case IV) & 70 & 5 & 41 \\
Pre-backoff (Case V) & 110 & 5 & 21 \\
\hline
\end{tabular}

show that the analytical model can accurately estimate the performance of group paging with pre-backoff under different $P_{P}$. In Figure 3, the cases with $W_{P B O} \neq 0$ always have lower $P_{C}$ and the case with the largest $W_{P B O}$ yields the lowest $P_{C}$. It shows that the collision probability of group paging with pre-backoff always decreases as $W_{P B O}$ increases. It is because pre-backoff spreads the UEs' first RA attempts to multiple RA slots.

Figure 4 shows the access success probability. It is found that the access success probability does not increase as $P_{C}$ decreases when $W_{P B O}$ increases. This is because a larger $W_{P B O}$ implies a smaller $N_{P T m a x}$ under the assumption of a fixed $I_{\max }$. From the analytical results of Figure $4, P_{S}$ starts to fall below 1 at a smaller value of $M$ in a larger $W_{P B O} . P_{S}=1$ can be maintained until $M=364,358$, and 92 for $W_{P B O}$ of 0,30 , and 150, respectively. Increasing $W_{P B O}$ may decrease $P_{S}$ for a small $M$ because $N_{P T m a x}$ is decreased. However, the increase in $W_{P B O}$ may increase $P_{S}$ for large $M$ since a larger pre-backoff window significantly reduces collision. Figure 4 shows that $P_{S}=69 \%$ can still be achieved for 1,000 UEs with $W_{P B O}=150$. In larger $M$, smaller $P_{P}$ increases $P_{S}$ since it limits the contending UEs to an RA slot.

Figure 5 shows the average access delay, $\overline{D_{A}}$. It can be seen from Figure 5 that group paging without pre-backoff is suitable for a group size less than 160 UEs, since $P_{S}=$ $100 \%$ can be attained with the minimum average access delay. Pre-backoff with $W_{P B O}=30$ becomes useful when $160<M<357$. In this region, the group paging with prebackoff can attain $P_{S}=100 \%$ with a lower average access delay. It is also found in Figure 5 that the average access delay is almost constant and is less dependent on $M$ for the cases of $W_{P B O}=150$. It is because the pre-backoff delay dominates the average access delay. It is also found that group paging with pre-backoff can significantly increase the access success probability while keeping a lower average access delay for the successful UEs when we have a big group size (e.g., $M=1,000$ ).

As indicated in Figures 3, 4, and 5, an effective value of $W_{P B O}$ is required to decrease $P_{C}$ within acceptable $\overline{D_{A}}$. Figure 6 demonstrates the tradeoff between $W_{P B O}$ and $W_{B O}$ under the same constraint of $N_{P T \max }=5$. It is found that the minimum $P_{C}$ is achieved when $W_{P B O}=150$ and $W_{B O}=21$. Among the cases, the highest value of $W_{P B O}$ is 150 whereas the highest value of $W_{B O}$ is 51 . We did not include the case where $W_{B O}=150$ (equal to the highest $W_{P B O}$ ) because it will have non-integer and too small value of $N_{P \text { Tmax }}$ to maintain the $I_{\max }=55$. Too-small value of $N_{P T \max }$ limits the RA attempt for each node, which will increase the total number of failed UEs. If higher $W_{B O}$ is implemented without the smaller $N_{P T \text { Tmax }}$, the group paging interval might become too long, which means a large value of $I_{\max }$. Therefore, increasing $W_{P B O}$ is more practical to decrease collision compared with increasing $W_{B O}$.

Figure 7 shows the optimal values of $W_{P B O}$ as a function of $M$ for $I_{\max }=55$. The optimal values are the smallest value of $W_{P B O}$ that yields the highest $P_{S}$ with the lowest $\overline{D_{A}}$ for a given value of $M$. Figure 7 depicts the relation between parameter settings and different scenarios of

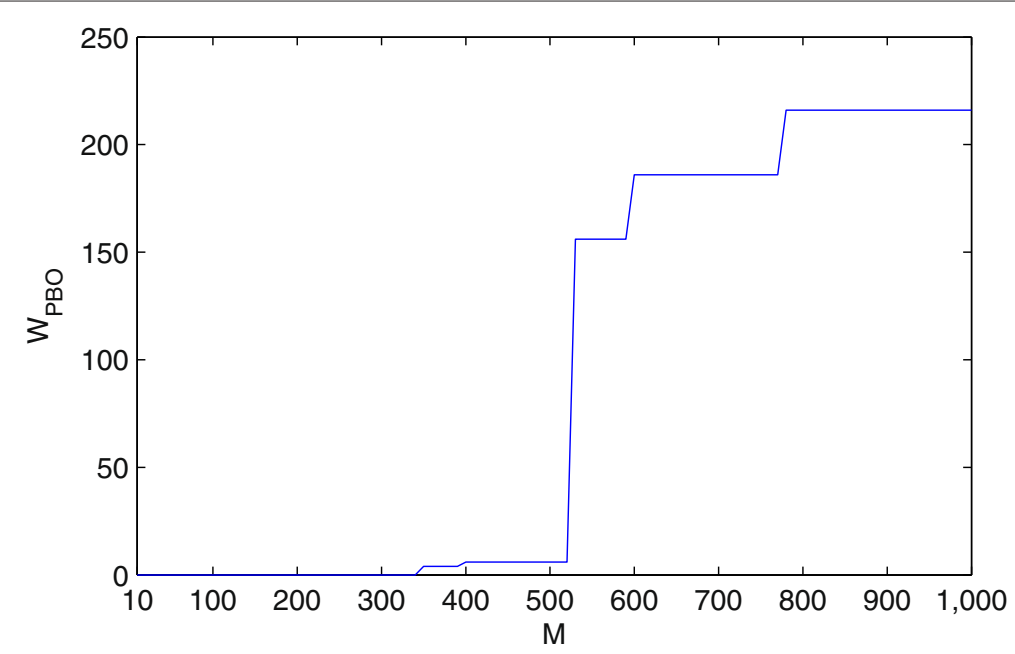

Figure 7 Optimal $W_{P B O}$ for each $M$ to reach the highest $P_{S}$ under $I_{\max }=55$. 
group size implementation. It suggests a value of $W_{P B O}$ to be used for a certain group size. After a value of $W_{P B O}$ is chosen, the setting of $N_{P \text { Tmax }}$ can be defined according to (1) for $I_{\max }=55$.

The simulation result shows that the proposed algorithm outperforms existing group paging when the group size is large. However, it is worthwhile to note that the result was obtained under an extreme assumption in which all the 54 preambles were reserved for group paging. In reality, the preambles are shared by all services and the network may only reserve five preambles for group paging. In this case, the proposed algorithm is expected to outperform the existing group paging when the group size exceeds 100 .

\section{Conclusions}

In this paper, we suggested enhancing the performance of group paging by enforcing UEs to perform pre-backoff before the first transmission of preamble. The application had to be delay tolerant, since the time for backoff window might be long. An analytical model was then presented to derive the performance of group paging with pre-backoff. Simulations were conducted to evaluate the group paging with pre-backoff in terms of collision probability, access success probability, the average access delay, and the tradeoffs with the existing backoff mechanism. The results verify the accuracy of the analysis and demonstrate that pre-backoff can greatly enhance the performance of group paging when the group size is big. The proposed analytical model can be used to determine the optimal pre-backoff window size under a target access success probability.

\section{Competing interests}

The authors declare that they have no competing interests.

\section{Authors' contributions}

This work was supported in part by the Ministry of Science and Technology, Taiwan, R.O.C., under Contract MOST 102-2221-E-011-003-MY3.

\section{Author details}

${ }^{1}$ Department of Electrical, Electronics and Computer Engineering, National Taiwan University of Science and Technology (NTUST), Taipei 106, Taiwan.

${ }^{2}$ School of Information Science and Technology, Southwest Jiaotong University, Chengdu, Sichuan 610031, China. ${ }^{3}$ Department of Electrical Engineering, University of Indonesia (UI), Depok 16424, Indonesia.

Received: 4 March 2014 Accepted: 2 February 2015

Published online: 20 February 2015

\section{References}

1. E Khorov, A Lyakhov, A Krotov, A Guschin, A survey on IEEE 802.11ah: An enabling networking technology for smart cities. Comp. Commun (2014). doi:10.1016/j.comcom.2014.08.008

2. R Harwahyu, RG Cheng, RF Sari, in International Symposium on personal Indoor and Mobile Radio Communications. Consecutive group paging for LTE networks supporting machine-type communications services (IEEE England, 2013), pp. 1619-1623. doi:10.1109/PIMRC.2013.6666401

3. 3GPP LG Electronics Inc. R2-104004 (2010). http://www.3gpp.org/ftp/ tsg_ran/WG2_RL2/TSGR2_70bis/Docs/R2-104004.zip. Accessed 12 Jan 2013
4. CH Wei, RG Cheng, SL Tsao, Performance analysis of group paging for machine-type communications in LTE networks. IEEE Trans. Vehicular Tech. 62, 7 (2013). doi:10.1109/TVT.2013.2251832

5. 3GPP ITRI R2-113198 (2010). http://wwW.3gpp.org/ftp/tsg_ran/ WG2_RL2/TSGR2_74/Docs/R2-113198.zip. Accessed 13 Jan 2013

6. CH Wei, RG Cheng, SL Tsao, Modeling and estimation of one-shot random access for finite-user multichannel slotted ALOHA systems. IEEE Commun. Lett. 16, 8 (2012). doi:10.1109/LCOMM.2012.060112.120376

7. AH Tsai, LC Wang, JH Huang, TM Lin, in Vehicular Technology Conference. Overload control for machine type communications with femtocells (IEEE Quebec, 2012), pp. 1-5. doi:10.1109/NTCFall.2012.6399236

8. H Tang, M Lei, X Wang, C Sun, in Business Computing and Global Informatization. The priority backoff algorithm and PUSCH power control for random access in TD-LTE trunking system (IEEE China, 2012), pp. 580-583. doi:10.1109/BCGIN.2012.157

9. G Farhadi, A Ito, in Vehicular Technology Conference. Group-based signaling and access control for cellular machine-to-machine communication (IEEE Las Vegas, 2013), pp. 1-6. doi:10.1109/NTCFall.2013.6692218

10. O Arouk, A Ksentini, Y Hadjadj-Aoul, T Taleb, in International Conference on Communications. On improving the group paging method for machine-type-communications (IEEE Sydney, 2014), pp. 484-489. doi:10.1109/ICC.2014.6883365

11. 3GPP ZTE R2-111916 (2011). http://www.3gpp.org/ftp/tsg_ran/ WG2_RL2/TSGR2_73bis/Docs/R2-111916.zip. Accessed 20 Dec 2014

12. W Jiang, X Wang, T Deng, in International Conference on Intelligent Green Building and Smart Grid. Performance analysis of a pre-backoff based random access scheme for machine-type communications (IEEE Taipei, 2014), pp. 1-4, doi:10.1109/IGBSG.2014.6835249

\section{Submit your manuscript to a SpringerOpen ${ }^{\circ}$ journal and benefit from:}

- Convenient online submission

Rigorous peer review

- Immediate publication on acceptance

- Open access: articles freely available online

- High visibility within the field

- Retaining the copyright to your article

Submit your next manuscript at $>$ springeropen.com 